\title{
Stroke Prevention-Surgical and Interventional Approaches to Carotid Stenosis
}

\author{
Kumar Rajamani • Seemant Chaturvedi
}

Published online: 7 June 2011

(C) The American Society for Experimental NeuroTherapeutics, Inc. 2011

\begin{abstract}
Extracranial carotidartery stenosis is an important cause of stroke that often needs treatment with carotid revascularization. To prevent stroke recurrence, carotid endarterectomy has been well-established for many years in treating symptomatic high- and moderate-grade stenosis. Carotid stenting is an appealing, less invasive alternative to carotid endarterectomy, and several recent trials have compared the efficacy of the 2 procedures in patients with carotid stenosis. Carotid artery stenting has emerged as an important mode of therapy for high-risk patients with symtomatic highgrade stenosis. This review focuses on the current data available that will enable the clinician to decide optimal treatment strategies for patients with carotid stenosis.
\end{abstract}

Keywords Carotid stenosis · Carotid endarterectomy Carotid stenting - Asymptomatic and symptomatic stenosis · High-risk patients

\section{Introduction}

Patients with ischemic stroke or transient ischemic attack (TIA) should be screened for internal carotid artery (ICA) stenosis. Large-vessel atherosclerotic disease accounts for approximately $20 \%$ of all ischemic stroke patients of which approximately half are due to extracranial carotid artery

Electronic supplementary material The online version of this article (doi:10.1007/s13311-011-0052-2) contains supplementary material, which is available to authorized users.

K. Rajamani $(\bowtie) \cdot S$. Chaturvedi

Comprehensive Stroke Program, Department of Neurology,

Wayne State University School of Medicine,

8C-UHC, 4201 St. Antoine,

Detroit, MI 48201, USA

e-mail:krajaman@med.wayne.edu stenosis. Patients with hemodynamically significant carotid stenosis should be considered for carotid revascularization, either the well-established surgical procedure of carotid endarterectomy (CEA) or carotid stenting.

For patients who have experienced recent carotid territory symptoms, CEA can be very effective in decreasing the long-term stroke risk if there is moderate-to-severe stenosis. Many patients without recent carotid territory symptoms (asymptomatic stenosis) also undergo CEA, although the benefit is less certain for this group of patients. With advances in medical therapy, the benefits of carotid revascularization for asymptomatic carotid stenosis have come under further scrutiny. Some patients with carotid stenosis are not ideal candidates for surgery due to medical comorbidities (e.g., severe heart or lung disease) or surgical anatomic factors (e.g., previous surgery or radiation to the neck), and are considered as "high risk for CEA." In this group of subjects, carotid artery stenting (CAS) is an alternative to CEA for stroke prevention. The role of CAS in conventional risk patients has been compared in several recent trials and perhaps may be on equal footing to CEA, but subgroups may be identified as to who benefits from one or the other based on patient characteristics. In this chapter, we shall review the current data pertaining to CEA and CAS for stroke prevention.

\section{Carotid Endarterectomy for Symptomatic Carotid Stenosis}

Before 1990, CEA had been used as a tool for stroke prevention for many decades without much certainty regarding its benefits. After 2 relatively unsuccessful attempts for a definitive answer to the clinical question of the value of CEA [1, 2], 2 large-scale randomized 
studieswere launched in the 1980s: the North American Symptomatic Carotid Endarterectomy Trial (NASCET) [3] and the European Carotid Surgery Trial (ECST) [4]. A third randomized study, the Veterans Affairs Cooperative Study [5], was stopped early for ethical reasons after the NASCET and the ECST reported a clear benefit in surgically treated patients.

\section{High-Grade Symptomatic Internal Carotid Artery Stenosis}

The NASCET and the ECST were pivotal studies that evaluated CEA in comparison with the best, prevalent medical therapy for prevention of ischemic stroke in patients with symptomatic carotid stenosis. Patients with ICA stenosis, determined by angiography and previous TIA,nondisabling ischemic stroke in the ipsilateral hemisphere, or retinal ischemic symptoms, were included in both randomized control trials. Both studies published an interim report in 1991 and a final report in 1998 [6], and both reports indicated a significant benefit with CEA in patients with high-grade stenosis (i.e., 70-99\% occlusion). Pooled analysis combining the 2 studies and data from the Veterans Affairs trial (VA309) found CEAwas associated with an absolute risk reduction (ARR) of $16 \%$ in the primary endpoint of ipsilateral ischemic stroke, perioperative stroke, or perioperative death at 5 years (with the number needed to treat (NNT) of 6.3) [7]. Disabling or fatal ipsilateral ischemic strokes, perioperative strokes, and perioperative deaths were reduced in the CEA arm by $7 \%$. The long-term findings of NASCET and the ECST trials emphasized the durability of stroke prevention achieved with CEA in patients with high-grade stenosis after more than 8 years of follow-up, as well as its efficacy in preventing both mild and disabling strokes.

\section{Moderate-Grade and Low-Grade Internal Carotid Artery Stenosis}

The NASCET study reported comparatively lessimpressive results for CEA vs medical therapy in patients with moderate carotid stenosis (30-69\%) than in patients with high-grade stenosis [6]. Among patients with less than 50\% stenosis, the risk of stroke after 5-years of follow-up did not significantly differ between the surgical treatment arm and the medical arm (14.9\% vs $18.7 \%)$. However, in patients with stenosis in the range of 50 to $69 \%$ (high tomoderate stenosis), the 5-year risk of ipsilateral stroke was $15.7 \%$ in the surgical group compared with $22.2 \%$ in the medical group (ARR, 6.5\%). Notably, in this group, CEA did not confer a benefit to women, patients with diabetes, nor those with previous TIA. Women with 50 to $69 \%$ stenosis were found to have a low risk of stroke on medical therapy, and consequently benefited from surgery only if they met the criteria for additional risk factors, such as age greater than 70 years, severe hypertension, history of myocardial infarction, or a hemispheric (as opposed to a retinal) event [8]. Women also had higher perioperative mortality than men. The influence of gender on benefit with CEA is discussed in more detailas follows.

In regard to patients with moderate stenosis, the ECST findings varied considerably from the NASCET study findings. Patients in the 30 to $49 \%$ and 50 to $69 \%$ stenosis groups, both categorized as moderategrade stenosis, did not receive major benefit with surgery. This difference in outcome between the 2 major trials is partially related to the different methods each trial used to estimate the degree of stenosis on carotid angiography. Careful review has shown that the method used in the ECST tended to overestimate the degree of stenosis compared with the NASCET method [9]. Hence, many of the patients with moderatestenosis, according to NASCET criteria, were classified as having high-grade stenosis in the ECST, and many patients with 50 to $69 \%$ stenosis included in the moderategrade stenosis group in the ECST would have been classified as having less than $50 \%$ stenosis in the NASCET. Clinically significant differences in the outcomes of the 2 trials, especially among this group of patients, were seen as a consequence of this difference in methodology. Rothwellet al. [10] reanalyzed the angiograms of patients studied in the ECST, according to the method of stenosis measurement used in the NASCET, and they demonstrated remarkable consistency in the results of both the severe and moderate stenosis groups in both trials (Table 1). In the pooled analysis of symptomatic patients with moderate 50 to $69 \%$ stenosis, there was an ARR of $4.6 \%(\mathrm{NNT}=22)$ in the surgical arm compared to medical therapy in the risk of ipsilateral stroke or perioperative stroke or death. Disabling and fatal ipsilateral strokes were also reduced by $2.3 \%$ [7]. Thus, in the moderate stenosis group, the benefit of surgery, although statistically significant, was marginal and not as robust as in the high-grade stenosis group.

In the 30 to $49 \%$ stenosis group, surgery was associated with an ARR for stroke or death of $1.3 \%$ compared with medical treatment $(P=0.6)$, and in the low-grade stenosis group $(<30 \%$ stenosis $)$, surgical treatment was actually harmful, increasing the risk of stroke and death (ARR $-3.6 \%$; $p=$ 0.007). Therefore, accurate measurement of carotid stenosis is critical in clinical decision-making. In their final report, the ECST authors recognized this fact by recommending that the NASCET method of measuring carotid stenosis be adopted as the standard (Fig. 1) [10].

The two large randomized clinical trials NASCET and ECST both used DSA for establishing the degree of carotid stenosis. Hence, this has become the gold standard for reference. However, DSA is an invasive procedure that requires injection of radiocontrast dye and carries a small, 
Table 1 Risk of ipsilateral stroke at 5 years after carotid endarterectomy compared with best medical therapy in NASCET and ECST

\begin{tabular}{llllll}
\hline Stenosis (\%) & \multicolumn{2}{l}{ Risk in NASCET (\%) } & \multicolumn{3}{c}{ Risk in ECST (\%) } \\
\hline & Medical & Surgical & ARR & Medical & Surgical \\
$70-99$ & 28.0 & 13.0 & 15.0 & 26.5 & 14.9 \\
$50-69$ & 22.2 & 15.7 & 6.5 & 9.7 & 11.1 \\
$<50$ & 18.7 & 14.8 & NS & 6.2 & 11.6 \\
\hline
\end{tabular}

ARR $=$ absolute risk reduction; ECST $=$ European Carotid Surgery Trial; NASCET $=$ North American Symptomatic Carotid Endarterectomy Trial; $\mathrm{NS}=$ nonsignificant

but definite risk of mortality and morbidity (0.5-3\%). Consequently, noninvasive or less invasive tests are increasingly being advocated. Carotid ultrasound imaging is noninvasive and this testing not only can reliably provide information on the degree of stenosis, but it can also provide the plaque morphology and its extent. However, carotid ultrasound imaging also has several limitations because it is operator dependent, and if this testing is not performed in a standardized and thorough manner, it is prone to errors. Heavy calcification can produce acoustic shadowing and then be misleading. More distal parts of the carotid artery behind the mandible may not be easy to insonate. Tortuous vessels can produce abnormalities, which can mimic stenosis.

Magnetic resonance angiography offers the advantage of a noninvasive 3-dimensional view of the carotid vessels. B (ecause of its high sensitivity $(87-95 \%)$ and lower specificity (44-88\%), it can be reliably used to rule out the presence of carotid disease. However, it may not reliably discriminate between complet eobstructions of the vessel from trickle flow, as it tends to overestimate stenosis. Metallic stents may also cause interference with image quality, as it is prone to artifacts [11]. Computed tomographic angiography is a quick and accurate method of

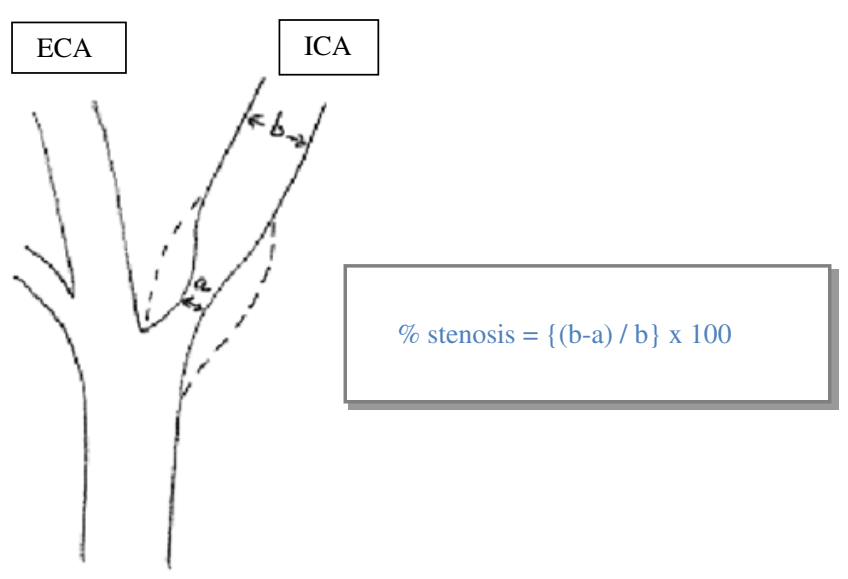

Fig. 1 The North American Symptomatic Carotid Endarterectomy Trial (NASCET) method of measuring degree of carotid stenosis on Digital Subtraction Angiography $(\mathrm{DSA})$. ECA $=$ External Carotid Artery; ICA = internal carotid artery determining carotid stenosis. Because of its speed, it is less prone to patient movement artifacts, and it is ideally suited for use in emergency settings. However, it does use ionizing radiation, and a fairly large bolus of radiocontrast dyecan be potentially nephrotoxic in individuals who are prone to the effects of it. Heavy calcification can result in inaccurate measurements of stenosis.

The decision to perform carotid revascularization often depends on accurate measurement of the degree of stenosis by 1 or 2 of these noninvasive tests, depending on the availability and local expertise. The DSA is reserved for complicated situations or when the initial evaluations are inconclusive or contradictory.

\section{Timing of Surgery}

The issue of proper timing of CEA aftera TIA or stroke has been greatly debated. Some are concerned that carotid surgery after a major cerebral infarction could result in adverse outcomes caused by cerebral hemorrhage $[12,13]$. However, in the NASCET trial, postoperative intracranial hemorrhage occurred in only $0.2 \%$ of patients and was nonfatal in each case [14]. Altered autoregulation and hyperperfusion in the ischemic vascular bed distal to the endarterectomy are probably responsible for these intracranial hemorrhages. Others have suggested that the use of antithrombotic agents in the perioperative and postoperative periods could be the cause of these types of hemorrhages [15].

In the past, concerns about postoperative hemorrhage often led to a delay in surgery for a few months after the initial ischemic event. However, a delay in surgeryexposes the patient to an excess risk of recurrent stroke in the interim period. Lovett et al. [16] have shown that the risk of stroke recurrence within the first month is high, especially in large-vessel disease. Another study estimated the risk of subsequent stroke after TIA to be approximately $10.5 \%$ at 3 months, with the majority of recurrent strokes occurring in the first week [17]. In theory, the risk of recurrence could recede in the months and years after the initial event, possibly as a result of healing or stabilization of the symptomatic plaques and development of adequate collateral blood vessels. 
In the pooled analysis of the symptomatic CEA trials, Rothwellet al. [18] have shown that CEA was not only safe, but was most beneficial when performed within 2 weeks of the index event [18]. Consequently, current treatment guidelines from the American Academy of Neurology, as well as the American Stroke Association (ASA) and the American Heart Association (AHA), recommend that CEA for patients with nondisabling strokes should be performed without delay and preferably within 2 weeks of the primary stroke $[19,20]$.

Overall, the ASA and AHA guidelines state that CEA is recommended by a surgeon with a stroke/death rate of $<6 \%$ for patients with severe stenosis (70-99\%) and a stroke or TIA in the territory of the stenosed vessel within the preceding 6 months (class I, level A recommendation). For patients with recent symptoms and 50-69\% stenosis, CEA is recommended, depending on factors such as age, gender, severity of symptoms, and medical comorbidities (class I, level A). For patients with $<50 \%$ stenosis, there is no evidence that CEA is useful [20].

\section{Which Patients Benefit Most from Carotid Endarterectomy?}

The multicenter CEA trials have led to several subgroup analyses of various clinical and radiologic features and their relationship to the benefits ofsurgery. Clinicians should recognize that even when performed by vetted surgeons, CEA is not a benign procedure. In randomized trials of symptomatic patients, the perioperative risk of stroke or death was approximately 7\% [7]. In fact, if this benchmark of safety cannot be achieved, the benefit of CEA provided to patients by way of stroke prevention is diminished. Hence, identifying the patients most at risk for recurrent events is vitally important to ensure that they receive maximum benefit. Many of the subgroup analyses should be viewed as exploratory because of potential group imbalances and limited statistical power. However, information from the pooled studies is more credible.

\section{Role of Gender: Men $v s$ Women}

Besides degree of stenosis and the timing of surgery, age greater than 75 years and male sex were statistically significant predictors of benefit in the pooled analysis of the endarterectomy trials [18]. It was observed that women on medical therapy had fewer recurrent events, but higherperioperative risk, resulting in a worse surgical risk/ benefit ratio compared to men. In a meta-analysis of all published studies between 1980 and 2004, women had a significantly higher risk of perioperative stroke and death than men (odds ratio, $1.31 ; p<0.001)$ [21]. The cause for this imbalance is unclear, but the smaller size of the carotid arteries in women, relative to men, is a possible explanation. Similar raised risks were described in another report combining data from the NASCET and the Aspirin and Carotid Endarterectomy (ACE) study [8]. The benefit from CEA was similar in women and men with high-grade ICA stenosis (5-year ARR, $15.1 \%$ vs 17\%, respectively), but women did indeed have a higher risk of perioperative stroke and death than men. Although men benefited from CEA in the moderate-stenosis group, there was no clear benefit in women with the same disease severity.

\section{Age}

Due to aging of the population, clinicians will increasingly encounter patients who are 80 years of age and greaterwho have carotid stenosis. The NASCET initially excluded patients in this age group, and although the ECST studied patients of any age, it is not clear how many patients in this age group were actually included. In a review of more than 2500 CEA procedures performed in octogenarians, the combined perioperative stroke and death rate was $3.45 \%$, which is within acceptable limits [22]. In another pooled analysis of trials of CEA for symptomatic stenosis in patients aged $>75$ years, benefit was higher compared to younger patients [21]. Administrative database studies have shown an increased perioperative mortality with increasing age; therefore, careful patient evaluation is mandatory when CEA is contemplated in octogenarians [23]. If an elderly symptomatic CEA candidate is medically fit, CEA should not be withheld. As benefit accrues during 1 to 2 years after surgery, these patients should ideally have a life expectancy that exceeds this period.

Symptoms at Presentation: Retinal vs Hemispheric Stroke

Risk of stroke recurrence can be stratified on the basis of symptoms at presentation. For example, transient visual symptoms resulting from carotid stenosis are more likely to be benign than serious. In the NASCET group, the risk of recurrent stroke among medically treated patients presenting with transient monocular blindness was significantly lower than in those presenting with hemispheric TIAs $(10 \%$ vs $20 \%$ in a timespan of 3 years) [24]. The risk of subsequent ischemic events was raised in individuals with transient monocular blindness treated medically if they had coexisting risk factors, including age greater than 75 years, symptomatic peripheral vascular disease, and 80 to $94 \%$ stenosis of the ICA without adequate collateral circulation. Consequently, among patients with transient monocular blindness, CEA was beneficial only when ICA stenosis $(>50 \%)$ was associated with these additional stroke risk factors. 


\section{Contralateral Internal Carotid Artery Occlusion}

Another factor that requires significant consideration when treating a patient with symptomatic carotid stenosis is contralateral ICA occlusion. Although some authors believethis conditiondoes not impact prognosis after CEA [25, 26], others have reported that it is associated with raised perioperative risk [27]. Gasecki et al. [28] described 43 patients in the NASCET database with contralateral ICA occlusion. They found the risk of perioperative stroke to be significantly higher in these patients than in those who had significant contralateral stenosis but were not occluded (14\% vs 5\%). However, the long-term outcome at 2 yearswas better in the surgery group than in the medical group ( $22 \%$ vs $69 \%$ risk of ipsilateral stroke). The authors concluded that there is significant benefit from CEA performed for symptomatic high-grade stenosis, even in the presence of contralateral ICA occlusion.

\section{Carotid Plaque Ulceration}

The pathophysiologic mechanisms of plaque ulceration and the potential for thrombosis and distal embolization have been extensively studied. After inspection of more than 1000 postoperative specimens after CEA, Park et al. [29] concluded that plaque ulceration is associated with symptomatic rather than asymptomatic plaques. Fisher et al. [30] confirmed this finding after careful study of samples collected from the NASCET study and the Asymptomatic Carotid Atherosclerosis Study (ACAS), which also showed that ulcerated plaques developed in the contralateral carotid artery as often as they developed in the ipsilateral symptomatic artery. In the NASCET study, although patients were not randomized prospectively on the basis of plaque ulceration, a post-hoc analysis revealed that the presence of ulceration, determined by angiography, significantly increased the risk of stroke in medically treated patients with severe stenosis by as much as 3 times [31]. However, these patientsare candidates for CEA because of the degree of stenosis alone. Moreover, detection of carotid plaque ulceration by bothcarotid duplex and angiography is currently unsatisfactory. In a study comparing surgical specimens with angiographic data in 500 patients from NASCET, angiography had a $45.9 \%$ sensitivity and $74 \%$ specificity with a positive predictive value of $71 \%$ for diagnosing plaque ulceration [32]. Future improvements in imaging technologies may allow more accurate identification of plaque ulceration and other plaque characteristics, which could result in more efficient stroke prevention. High-resolution magnetic resonance imaging (MRI) of atherosclerotic plaques has been used to determine the composition of plaques with a high degree of reliability. Besides ulceration, additional features of a "vulnerable plaque," such as intraplaque hemorrhage, lipid-necrotic core, and fibrotic calcified caps can be accurately identified. Biological processes, such as inflammation and neovascularization are capable of being identified by MRI. This not only could allow screening for high-risk patients, but it could also make it possible for early treatment initiation and noninvasive monitoring $[33,34]$.

\section{Carotid "Near Occlusion"}

When using catheter angiography to assess severe carotid stenosis, the flow in the distal ICA beyond the stenosis is occasionally reduced and seems to be "collapsed." These patients are classified as having "near occlusion." The diagnosis of near occlusion is made by the delayed appearance of contrast in the ipsilateral intracranial ICA compared with the external carotid artery and a smaller diameter of the ICA compared with the external carotid artery. The contrast is diluted because of the collateral circulation. Morgenstern et al. [35] identified 7.6\% of the NASCET population as having carotid near occlusion and observed that the risk of stroke recurrence in this group was significantly less than that in the 90 to $94 \%$ stenosis group (11\% vs 35\%). The ARR of stroke in the CEA-treated group with near occlusion was $7.9 \%$ compared to the medically treated group. Using combined NASCET and ECST datasets, Fox et al. [36] identified subsets of patients with near occlusion; the risk of stroke in the medically treated arm in this group was $15.1 \%$ compared with $10.9 \%$ in the surgical arm (ARR, 4.2\%). The reason for the low risk of stroke in this group is unclear, but it could due to good collateral circulation from the opposite side or the ipsilateral external carotid artery. However, as acknowledged by the authors, the sample size and event rates were too small to make definitive conclusions. CEA can be considered in these patients, although the benefit is muted.

\section{Carotid Endarterectomy for Asymptomatic Carotid Stenosis}

The role of CEA in asymptomatic individuals is much less certain and still much debated. The ACAS [37] and the Asymptomatic Carotid Surgery Trial (ACST) [38] are large studies that have investigated this issue.

In the ACAS, patients were enrolled to receive either best medical treatment or medical therapy plusendarterectomy if they had stenosis greater than $60 \%$, but were otherwise healthy [37]. The study was stopped early after 2.7 years of average follow-up. In the surgical arm, the recurrent combined event rate for ipsilateral stroke, any perioperative stroke, and death at 5 years was projected to be $5.1 \%$, compared with $11 \%$ in the medical arm, which 
wasa relative risk reduction of $55 \%$ and an ARR of $5.9 \%$. The marginal benefit with surgery could be a result of the exceptionally low perioperative risk of $1.5 \%$ achieved in the trial. Whether this low perioperative stroke rate can be uniformly achieved in "real life" situations is doubtful. For example, in a study of over 1800 asymptomatic CEA cases from Ontario, the perioperative stroke and death rate was $4.7 \%$ [39].

Although it is frequently reported that the ACST findings were similar to those of the ACAS, there were important differences in the 2 study designs. In the ACAS, the primary analysis compared strokes occurring in the territory of the operated carotid artery, whereas the ACST included strokes in any vascular territory. In addition, conventional angiography was not mandated for either group in the ACST. After 5-years of follow-up, the risk of recurrent stroke for the surgical group in the ACST was $6.4 \%$ and $11.8 \%$ for those on medical treatment, respectively [37]. This difference ( $13.4 \%$ vs $17.9 \%$ with a net benefit of $4.5 \%$ ) was more or less evident, even after 10 years [40]. The risk of perioperative stroke or death was $2.8 \%$. Importantly, this study showed a significant reduction of fatal or disabling strokes in the surgical arm $(3.5 \%$ vs $6.1 \%$ in the medically treated group; ARR, 2.6\%; $p<$ 0.004). Approximately half of all ipsilateral recurrent strokes that occurred were classified as fatal or disabling. The ACAS showed a trend toward reduction in fatal and disabling strokes with surgery, but it did not reach statistical significance (ARR, 2.7\%; $p=0.26$ ). There was no clear benefits of CEA in the patients age 75 years and older in the ACST.

A meta-analysis of data from 5223 patients from 3 major trials of CEA for asymptomatic carotid stenosis was performed by Chambers and Donnan [41]. Surgery conferred a significant benefit in terms of the composite primary outcome (i.e., any perioperative or subsequent stroke, and all-cause perioperative mortality; relative risk, $0.69 ; 95 \%$ CI $0.57-0.83$ ). The overallrisk of perioperative stroke or death was $2.9 \%$. Subgroup analysis revealed men received more benefit from surgery than did women, and younger patients benefited more than older patients. Unlike the symptomatic stenosis trials, stenosis severity did not correlate with a benefit from surgery. Despite these findings, some have argued against the routine use and widespread enthusiasm for CEA in asymptomatic patients. Barnett et al. [42] highlight that the absolute annual risk reduction of stroke in this asymptomatic group is approximately $1 \%$, with a number needed to treat (NNT) of 83 [42]. Moreover, it has been estimated that approximately half the strokes in asymptomatic individuals are not related to the stenosed carotid artery, but they are rather lacunar strokes or caused by cardioembolicevents [43].
As previously discussed, the benefit of surgery in patients with carotid stenosis is highly dependent on perioperative stroke risk. A low perioperative stroke risk is especially critical for asymptomatic patients in whom the marginal benefit can be lost if the risk is not within recommended limits. Therefore, practicing clinicians must be aware of the local and institutional complication rates to advise patients. In a study of 12 academic centers and 1160 procedures, Goldstein et al. [44] reported a perioperative risk of stroke or death of $2.8 \%$. Notably, the rate was higher in symptomatic than in asymptomatic individuals. Postoperative stroke and death was also significantly raised in women, older individuals ( $>75$ years), those with associated congestive heart failure, and those undergoing simultaneous coronary artery bypass grafting surgery. Thus, the American Academy of Neurology guidelines recommend that CEA for asymptomatic stenosis be considered only for patients aged 40 to 75 years, with at least a 5 -year life expectancy. In addition, the surgeon's complication rate should be reliably documented as less than $3 \%$ [19].

In the last 15 years, the recognition of the role of early and comprehensive medical management of cerebrovascular disease has led to a great but highly underappreciated reduction of stroke risk in this population of patients. There is paucity of data as to the exact annual risk of stroke in patients with asymptomatic carotid stenosis on modern medical therapy. By 1 estimate, the annual risk of stroke has dropped significantlyto $<1 \%$ per year with medical therapy alone, raising serious questions as to the benefit of any revascularization procedure [45]. Spence et al. [46] have shown that transcranial Doppler can identify a subgroup of patients with asymptomatic stenosis who have microembolic signals that are at higher risk for stroke than those who do not have these microembolic signals. The risk of stroke in patients with asymptomatic stenosis, but without microembolic signals, is remarkably low. They further demonstrate that intensive medical therapy of arterial plaques can reduce the number of patients with microembolic signals by $90 \%$ and that revascularization procedures should be considered only in the small minority who can be demonstrated to be at high risk [47].

Guidelines from the ASA and AHA indicate that patients with asymptomatic stenosis should be screened for other treatable causes of stroke and that intensive treatment of stroke risk factors should be pursued (class I, level C) [48]. In addition, the use of aspirin is recommended in subjects with asymptomatic stenosis. CEA is recommended only in highly select patients with high-grade stenosis, and the surgeon should have a stroke/death rate of $<3 \%$ (class I, level A). There should be a thorough understanding of the goals of the procedure, the patient's life expectancy and comorbidities, and patient preferences. 


\section{Perioperative Drug Therapy}

The NASCET investigators initiallyobserved that patients receiving low-dose aspirin ( $0-325 \mathrm{mg} /$ day $)$ in the perioperative period had a higher risk of perioperative stroke and death than those on higher doses (650-1300 mg/day). This observation led to the randomized Aspirin and Carotid Endarterectomy (ACE) trial [49], which found that perioperative stroke or vascular death risk in the low-dose aspirin (81-325 mg/day) arm was $6.2 \%$ compared with $8.4 \%$ in the high-dose arm (650-1300 mg/day), a finding contrary to the previous observation. A more recent systematic review of all trials has attempted to address the question of optimum anti-platelet therapy during CEA for symptomatic and asymptomatic carotid stenosis [50]. This study found that perioperative stroke risk among those receiving anti-platelet agents was significantly reduced, but that the risk of perioperative death was not significantly altered. The findings also indicated that anti-platelet agents could increase the risk of hemorrhage. The widespread belief that anti-platelet agents reduce the risk of nativevessel or graft thrombosis and myocardial infarction after vascular surgery (including CEA), however, means that most clinicians use anti-platelet therapies in the perioperative period for patients undergoing CEA. Based mostly on the ACE trial, aspirin ( 81 to $325 \mathrm{mgs}$ per day) rather than higher doses (650 mg or $1300 \mathrm{mg}$ per day) is recommended during CEA in both symptomatic and asymptomatic patients. Aspirin should be commenced prior to surgery and continued for a minimum of 3 months after surgery, and has been shown to reducethe risk of perioperative stroke, myocardial infarction, and death. There is insufficient data for specific recommendations for use of the other antiplatelet agents.

Evidence that statins [51] and beta blockers [52] reduce morbidity and mortalitywhen used during vascular surgery is mounting. McGirtet al. [53] reported that use of statins, compared with absence of statin treatment, during CEA significantly reduced the risk of perioperative stroke $(1.2 \%$ vs $4.5 \% ; p<0.01)$ and death $(0.3 \%$ vs $2.1 \% ; p<0.01)$. These observations are intriguing, but more definitive studies are needed before broad recommendations for routine use of these medications can be advocated in the perioperative period.

\section{The Risks Associated with Carotid Endarterectomy}

The risks of surgery should be carefully discussed with patients before CEA. Risks include perioperative ischemic stroke, hemorrhagic stroke, cranial nerve injury, myocardial infarction, congestive heart failure, and neck hematoma with consequent airway compromise. Perioperative ische- mic stroke occurs as a result of thrombotic occlusion of the operative site, distal thromboembolism of debris from the operative site, cross clamping of the ICA, or a combination of these factors. Ischemic stroke usually occurs within the first 12 to $24 \mathrm{~h}$ after surgery, but it can also occur later in recovery. If a patient wakes up from anesthesia with a deficit or develops one soon thereafter, emergent exploration of the operative site for thrombosis and consequent occlusion or other correctable operative defects is usually undertaken. Carotid duplex imaging or carotid angiography can be performed with a view to identifying occluded vessels.A computed tomographic scan of the brain is probably less useful, because intracranial hemorrhage is rare after CEA. The advantage of computed tomographic angiography or emergent DSA, when available, is their ability to visualize distal intracranial vessels occlusions in addition to patency the internal carotid artery. Further management options could include intra-arterial thrombolysis [54], as well as emergency stenting of the carotid artery [55]. However, the benefit of reoperation cannot always be predicted. Of the 10 patients who underwent reoperation in the NASCET, none demonstrated any benefit [14]. Furthermore, Findlay and Marchak [56] reported that 13 of 24 patients had postoperative strokes after CEA and underwent emergency reoperations [56], yet only 4 of these patients were reported to show any benefit.

Fortunately, hemorrhagic stroke is rare. Only $0.2 \%$ of the NASCET cohort was reported to have this type of stroke. In a retrospective review of patients undergoing CEA, Piepgras et al. [13] found this complication occurred in $0.6 \%$ patients, mainly in those with hypertension.

Severe carotid stenosis with limited collateral flow could result in postoperative hyperperfusion syndrome, which has been reported in 0 to 3\% after CEA [57]. Clinical features typically include ipsilateral headache, seizures, and focal neurological deficits in the setting of hypertension after CEA. Diminished cerebrovascular reserve and hypertension both contribute to hyperperfusion and consequent cerebral edema. The prognosis is often grave if not recognized early during surgery. There is an increase in the cerebral blood flow, which is often more than $100 \%$ of baseline levels. Treatment strategies are directed towardregulation of blood flow, which is dependent on the elevated blood pressure. Labetolol and clonidine are drugs of choice, whereas nitroprusside, glyceryltrinitate, angiotensin-converting enzyme inhibitors, and calcium channel blockers are to be avoided because of their cerebral vasodilating properties. Cerebral edema should be treated when present with proper head positioning ( $30^{\circ}$ elevation), sedation, and administration of mannitol or hypertonic saline. When seizures occur, anticonvulsants are administered, but prophylactic use of thesedrugs are probably not useful. Wound complications, such as infections and hematoma occurred in $9.3 \%$ of patients in the 
NASCET. Wound hematoma is of particular concern, because in the NASCET it was associated with raised perioperative stroke risk $(14.5 \%$ vs $5.9 \%$ in patients without hematoma) [14]. Large hematomas can also result in airway compromise, requiring immediate evacuation. Smaller hematomas can be managed expectantly and more conservatively. Cranial nerve injuries include those to the hypoglossal nerve, vagus nerve, or branches of the facial nerveand occur in $8.6 \%$ of patients, but are commonly transient and mild.

Overall, the risk of complications with CEA is raised in symptomatic patients, in patients with contralateral ICA occlusion, in patients with hemispheric rather than retinal ischemic events, inpatients aged 75 years or more, in patients who are women, and in patients undergoing reoperation [44, 58, 59]. Severe systemic illnesses, such as congestive heart failure, severe respiratory insufficiency, uncontrolled hypertension, and angina are contraindications to CEA.

\section{Carotid Angioplasty and Stenting}

In the past 10 to 15 years, the carotid angioplasty and stenting (CAS)procedure has attracted increased attention as a less invasive alternative to CEA. The CAS procedure has continued to evolve for years in terms of operator experience, as well as technological advances [60]. With improving results, the CAS procedureclearly has the potential to be considered as frontline therapy for at least some patients with carotid stenosis. Distal thromboembolism has been an important cause of complications during the stenting procedure. To minimize complications from embolism, distal embolism protection devices (EPD) have been advocated. In the US, the EPD device use has been mandated by the Center for Medicare and Medicaid Services (CMS) for reimbursement, as well as in major trials. Unfortunately, none of the EPDs can completely prevent all embolic events. Proximal EPDs have recently been used with some success with the rationale that the bulky EPD does not cross the stenosis, making it less likely to dislodge thrombi from the plaque. In spite of technolog- ical advances, the indications for performing this procedure are still being debated.

\section{CAS in "High-Risk"Patients}

Previously discussed trials of CEA, such as NASCET and ACAS, excluded patients who were at high risk for perioperative mortality and morbidity, and these patients had substantially worse outcomes than those reported in the trials [23, 61]. Patients at "high risk" for CEA have been treated with CAS as part of either industry-supported registries or randomized trials. Commonly used criteria for "high risk" CEA candidates are delineated in Table 2.

One randomized study, the Study of Angioplasty with Protection in Patients at High Risk for Endarterectomy (SAPPHIRE) [62] included both symptomatic and asymptomatic patients (close to $70 \%$ asymptomatic) with ICA stenosis who were judged to be high risk for CEA. Patients were randomly assigned to CEA or CAS. In the study population as a whole, the investigators concluded that CAS with distal emboli protection was not inferior to CEA in high-risk patients. The 30-day risk of stroke, death, or myocardial infarction was $4.4 \%$ in the CAS group compared to $9.8 \%$ in the CEA group. At 1-year followup, the combined rate of stroke, death, and myocardial infarction was significantly lower in those randomized to CAS compared to those getting CEA (12\% vs 20\%). Moreover, a second revascularization procedure was required significantly less often in the CAS group compared to the CEA group (0.6 \% vs 4.3\%). Most of the difference in the SAPPHIRE endpoint rates was due to the lower risk of non-Q wave myocardial infarction (MI) events in the CAS cohort.

Information on the 3-year outcome of patients in the SAPPHIRE has been reported, although follow-up was incomplete (with only $78 \%$ of patients who had 3-year data) [63]. For the outcome of periprocedure (within 30 days) stroke, MI, or death, or ipsilateral stroke between 31 to 1080 days, there was not a significant difference in the outcome in the CEA and CAS groups. There were $74 \%$ of CAS subjects and $70 \%$ of CEA patients free of this endpoint at 3 years. The relatively high 3 -year death rate in
Table 2 Commonly cited criteria determining "high-risk" for CEA

$\mathrm{CABG}=$ coronary artery bypass grafting; $\mathrm{CEA}=$ carotid endarterectomy; $\mathrm{CHF}=$ congestive heart failure; $\mathrm{COPD}=$ chronic obstructive pulmonary disease; $\mathrm{EF}=$ ejection fraction; $\mathrm{MI}=$ myocardial infarction

\begin{tabular}{ll}
\hline Medical & Surgical/Anatomical \\
\hline Left ventricular $\mathrm{EF}<30 \%$ & Contralateral carotid occlusion \\
Age $\geq 80$ years & Prior radiation to neck \\
Recent $\mathrm{MI}(\leq 30$ days $)$ & Open tracheostomy \\
Class III/IV angina or CHF & High cervical bifurcation \\
Severe COPD & Low/thoracic bifurcation \\
Need for CABG in $<30$ days & Contralateral recurrent laryngeal nerve palsy \\
Significant renal failure & Prior ipsilateral carotid endarterectomy \\
\hline
\end{tabular}


both groups, averaging $22 \%$, is concerning and raises questions as to the value and necessity of either procedure in a high-surgical risk cohort. The majority of the deaths were cardiac in nature, whereas neurological complications were responsible for deaths in only a very small number. More meaningful subgroup analysis was not possible because of the small numbers. In this study, although patient randomization was conducted by an expert panel in each center, it is possible that others may choose medical therapy over intervention in this high-risk group.

There have been numerous single-center case series and registry publications reporting results of CAS studies, which are performed as part of the mandated Food and Drug Administraton (FDA) postmarketing surveillance that has provided important insights into patient and physicianrelated features impacting outcomes. The periprocedure rate 30-day risk of stroke, death, and myocardial infarction has been between $1 \%$ to $8 \%$ [64]. In industry-sponsored registries, the 30-day combined risk of stroke, death, and MI has varied from $3.8 \%$ to $8.6 \%$ [64].

Predominantly based on the previously cited information, the FDA has approved the usage of stenting systems (Abbott Vascular Acculink/Accunet \& the AbbottXact/ Embolishield CAS systems, Illinois, USA.) for limited applications in treatment of carotid artery disease. The CMS currently reimburses treatment with the approved devices for symptomatic high-risk patients with only $>70 \%$ stenosis. Symptomatic patients with 50 to $69 \%$ stenosis and asymptomatic patients with $>80 \%$ stenosis will be reimbursed, only if treated under the setting of an approved clinical trial or registry.

The AHA/ASA guidelines state that in patients with symptomatic stenosis of $>70 \%$ in whom the stenosis is difficult to access surgically or with significant medical comorbidities, CAS in not inferior to CEA and can be considered (class IIb, level B). CAS practitioners should have a periprocedural stroke/death rate of $<4$ to $6 \%$ (class IIa, level B) [20].

\section{CAS in "Traditional-Risk" Patients}

Several recent randomized controlled trials of CAS compared to CEA in traditional risk patients have been published. The Stent-Supported Percutaneous Angioplasty of the Carotid Artery vs Endarterectomy (SPACE) [65] trial analyzed 1183 symptomatic patients who were randomized to either CAS or CEA. The 30-day risk of ipsilateral stroke or death was $6.84 \%$ for the CAS group compared to $6.34 \%$ in the CEA group, and the study could not prove noninferiority of the stenting procedure. At 2 years, the risk of the primary outcome in this study (ipsilateral stroke for more than 2 years, or any perioperative stroke or death) was similar in both groups. The study found an excess risk of carotid re-stenosis in the CAS group, although most were asymptomatic [66]. A similar study, the Endarterectomy vs Angioplasty in Patients with Symptomatic Severe Stenosis (EVA-3S) [67], was stopped earlier than planned for futility and safety. The 30 -day rate of stroke and death was 3.9\% in the CEA group compared to $6.1 \%$ in the CAS group. This discrepancy was significant and persisted after 6 months as well as at 4 years [68]. The authors concluded that widespread use of CAS is not justified in this group of patients. The 4-year analysis of this study showed that the differences in outcomes were largely due to periprocedural outcomes, whereas the risk of subsequent ipsilateral strokes were similarly low in both groups.

There was criticism of both these trials because of limited training of the interventionalists, multiple device types used (some without embolic protection) often with minimal training, and lack of standardized medical therapy [69]. For example, in the postmarketing Carotid ACCULINK/ACCUNET Post Approval Trial to Uncover Rare Events (CAPTURE 2) clinical study, there was an inverse relationship between the outcomes event rates and individual operator volume of experience [68]. A threshold of 72 cases was determined by the authors to be consistently associated with the American Heart Association defined rates of periprocedural complications.

The National Institutes of Health-supported Carotid Revascularization Endarterectomyvs Stenting Trial (CREST) recruited patients with symptomatic $(>70 \%$ stenosis by ultrasound or $>50 \%$ by angiography) and asymptomatic (70-99\%) stenosis. The primary endpoint (stroke, MI and perioperative death, and ipsilateral stroke after an average follow-up of 2.5 years) were similar in the 2 groups $(7.2 \%$ in the stenting arm $v s \quad 6.9 \%$ in the CEA arm). They

Table 3 Status of carotid stenting according to patient profile

- Symptomatic high-risk patients with 70-99\% stenosis can be considered for CAS

- Symptomatic high-risk patients with 50-69\% (moderate) stenosis should be offered CAS only in the setting of an approved clinical trial or registry

- Asymptomatic high-risk patients with $>80 \%$ stenosis should be offered CAS only in the setting of an approved clinical trial or registry

- Role for CAS in conventional risk patients with symptomatic $>50 \%$ is evolving. This should be avoided in patients 70 years and older with tortuous and calcified arteries. In younger patients, especially males, CAS is a reasonable option.

CAS $=$ carotid artery stenting 
reported a slightly elevated, but significant, 30-day risk of perioperative stroke in the stenting arm $(4.1 \%$ vs $2.3 \%)$, where as significantly more patients had developed perioperative $\mathrm{MI}$ in the CEA arm (1.1\% vs $2.3 \%)$. Although some have argued that this suggests the 2 procedures are equivalent, others have pointed out that strokes result in greater impairment in quality of life compared to MI, and consequently current stenting procedures could result in more harm. The CREST analysis showed no difference based on gender or symptom status. Age produced a significant effect on the outcomes, with a cutoff at approximately 70 years. Patients less than 70 years fared better with CAS, and those older fared better with CEA. This is contrary to what one might intuitively expect (i.e., CAS being a less invasive procedure would be better suited for older patients). It is likely the more tortuous and atherosclerotic calcified vessels in older patients that results in more strokes possibly from the introduction of the embolic protection devices. Moreover, periprocedural risk of events (stroke, MI, or death) was higher in women who underwent carotid stenting compared to CEA $(6.8 \%$ vs $3.8 \%$; $p=0.04)$. This difference was not found in men and may need to be considered when deciding therapy in women [70].

The International Carotid Stenting Study (ICSS) [11] was a multicenter study comparing CEA to CAS in symptomatic patients. The interim report on safety analysis showed that $8.5 \%$ risk of stroke, death, and MI in the CAS group compared to $5.2 \%$ in the CEA group. Moreover, an MRI substudy [71] of the ICSS revealed presence of 3 times more new ischemic lesions in the stenting group compared to CEA group. The study hence concluded that CEA should remain the treatment of choice in these patients until the long-term results were available. The ICSS had important differences from CREST, which could have contributed to the differing outcomes. ICSS included only symptomatic patients, and interventionists underwent a less stringent vetting procedure, both of which could possibly result in poorer outcomes in the CAS arm.

Large comprehensive meta-analyses of trails comparing CAS and CEA have been recently published [71, 72]. These provide good statistical evidencefor a $20 \%$ relative risk increase of periprocedure stroke or death and ipsilateral stroke with CAS; there is a $15 \%$ relative risk reduction in periprocedure MI compared to CEA. The increase in recurrent stroke rate was predominantly due to nondisabling strokes. There was no significant difference between the two groups in the risk of disabling strokes $(3.2 \%$ vs $2.8 \% ; p=0.18)$ nor of fatal strokes $(0.85$ vs $0.4 \%$; $p=0.11$ ). Moreover, the risk of long-term stroke events was significantly more in patients $>68$ years [71]. An overview of current CAS recommendations can be found in Table 3.

\section{Conclusions}

CEA underwent a resurgence in the 1990s after the landmark clinical trials demonstrated its benefit in carefully selected patient populations for secondary, and to a lesser extent primary, stroke prevention. This procedure prevents stroke in symptomatic patients with high-grade and moderate-grade ICA stenosis of more than $50 \%$. In asymptomatic patients with high-grade stenosis, the benefit is less and highly sensitive to the periprocedure stroke risk. "High-risk" patients, such as those with comorbid medical conditions, should be considered for CAS if they have high-grade symptomatic stenosis. Those high-risk patients with moderate-grade symptomatic or with asymptomatic stenosis $>80 \%$ may be considered for CAS only in the setting of a clinical trial or registry. It remains unclear if any revascularization procedure is necessary in asymptomatic patients who are at high-surgical risk. For conventional risk patients with carotid stenosis, CAS is emerging as a viable alternative, but CEA still seems superior because of lesser rates of perioperative and long-term stroke risks. But in certain subgroups, such as patients younger than 68 years, especially in males, the risks of CAS may be comparable to that of CEA. The FDA has approved of CAS for conventional risk patients, although the CMS does not reimburse yet for this indication. Documentation and dissemination of the institutional complication rates for both CEA and CAS is important to guide both the patient and the referring physician.

Acknowledgment Full conflict of interest disclosure is available in the electronic supplementary material for this article.

\section{References}

1. Fields WS, Maslenikov V, Meyer JS, Hass WK, Remington RD, Macdonald M. Joint study of extracranial arterial occlusion. V. Progress report of prognosis following surgery or nonsurgical treatment for transient cerebral ischemic attacks and cervical carotid artery lesions. JAMA 1970;211:1993-2003.

2. Shaw DA, Venables GS, Cartlidge NE, Bates D, Dickinson PH. Carotid endarterectomy in patients with transient cerebral ischaemia. J NeurolSci 1984;64:45-53.

3. Collaborators NASCET. Beneficial effect of carotid endarterctomy in symptomatic patients with high grade stenosis. NEJM 1991;325:445-453.

4. ECSTTrialists' Group. MRC European Carotid Surgery Trial: interim results for symptomatic patients with severe (70-99\%) or with mild $(0-29 \%)$ carotid stenosis. European Carotid Surgery Trialists' Collaborative Group. Lancet 1991;337:1235-1243.

5. Mayberg MR, Wilson SE, Yatsu F, et al. Carotid endarterectomy and prevention of cerebral ischemia in symptomatic carotid stenosis. Veterans Affairs Cooperative Studies Program 309 Trialist Group. JAMA 1991;266:3289-3294.

6. Barnett HJM, Taylor DW, Eliasziw M, et al. Benefit of carotid endarterectomy in patients with symptomatic moderate or severe stenosis. N Engl J Med 1998;339:1415-1425. 
7. Rothwell PM, Eliasziw M, Gutnikov SA, et al. Analysis of pooled data from the randomised controlled trials of endarterectomy for symptomatic carotid stenosis. The Lancet 2003;361:107.

8. Alamowitch S, Eliasziw M, Barnett HJM, for the North American Symptomatic Carotid Endarterectomy Trial and the ASAaCETG. The Risk and Benefit of Endarterectomy in Women With Symptomatic Internal Carotid Artery Disease. Stroke 2005;36:27-31.

9. Rothwell PM, Gibson RJ, Slattery J, Sellar RJ, Warlow CP. Equivalence of measurements of carotid stenosis. A comparison of three methods on 1001 angiograms.European Carotid Surgery Trialists' Collaborative Group. Stroke 1994;25:2435-2439.

10. Rothwell PM, Gutnikov SA, Warlow CP. Reanalysis of the final results of the European Carotid Surgery Trial. Stroke 2003;34:514-523.

11. Jaff MR, Goldmakher GV, Lev MH, Romero JM. Imaging of the carotid arteries: the role of duplex ultrasonography, magnetic resonance arteriography, and computerized tomographic arteriography. Vasc Med 2008;13:281-292.

12. Bernstein M, Fleming JF, Deck JH. Cerebral hyperperfusion after carotid endarterectomy: a cause of cerebral hemorrhage. Neurosurgery 1984;15:50-56.

13. Piepgras DG, Morgan MK, Sundt TM Jr., Yanagihara T, Mussman LM. Intracerebral hemorrhage after carotid endarterectomy. J Neurosurg 1988;68:532-536.

14. Ferguson GG, Eliasziw M, Barr HWK, et al. The North American Symptomatic Carotid Endarterectomy Trial: surgical results in 1415 patients. Stroke 1999;30:1751-1758.

15. Hafner DH, Smith RB 3 rd, King OW, et al. Massive intracerebral hemorrhage following carotid endarterectomy. Arch Surg 1987; 122:305-307.

16. Lovett JK, Coull AJ, Rothwell PM. Early risk of recurrence by subtype of ischemic stroke in population-based incidence studies. Neurology 2004;62:569-573.

17. Johnston SC, Gress DR, Browner WS, Sidney S. Short-term prognosis after emergency department diagnosis of TIA. JAMA 2000;284:2901-2906.

18. Rothwell PM, Eliasziw M, Gutnikov SA, Warlow CP, Barnett HJM. Endarterectomy for symptomatic carotid stenosis in relation to clinical subgroups and timing of surgery. The Lancet 2004;363:915.

19. Chaturvedi S, Bruno A, Feasby T, et al. Carotid endarterectomy an evidence-based review: Report of the Therapeutics and Technology Assessment Subcommittee of the American Academy of Neurology. Neurology 2005;65:794-801.

20. Sacco RL, Adams R, Albers G, et al. Guidelines for prevention of stroke in patients with ischemic stroke or transient ischemic attack: astatement for healthcare professionals from the American Heart Association/American Stroke Association Council on Stroke: co-sponsored by the Council on Cardiovascular Radiology and Intervention: The American Academy of Neurology affirms the value of this guideline. Stroke 2006;37:577-617.

21. Bond R, Rerkasem K, Cuffe R, Rothwell PM. A Systematic review of the associations between age and sex and the operative risks of carotid endarterectomy.CerebrovascDis 2005;20:69-77.

22. Grego F, Lepidi S, Antonello M, et al. Is carotid endarterectomy in octogenarians more dangerous than in younger patients? J CardiovascSurg (Torino) 2005;46:477-483.

23. Wennberg DE, Lucas FL, Birkmeyer JD, Bredenberg CE, Fisher ES. Variation in carotid endarterectomy mortality in the Medicare population: trial hospitals, volume, and patient characteristics. JAMA 1998;279:1278-1281.

24. Benavente O, Eliasziw M, Streifler JY, et al. Prognosis after transient monocular blindness associated with carotid-artery stenosis. N Engl J Med 2001;345:1084-1090.

25. Grego F, Antonello M, Lepidi S, et al. Is contralateral carotid artery occlusion a risk factor for carotid endarterectomy? Ann VascSurg 2005;19:882-889.
26. AbuRahma AF, Robinson P, Holt SM, Herzog TA, Mowery NT. Perioperative and late stroke rates of carotid endarterectomy contralateral to carotid artery occlusion: results from a randomized trial. Stroke 2000;31:1566-1571.

27. Klijn CJ, van Buren PA, Kappelle LJ, et al. Outcome in patients with symptomatic occlusion of the internal carotid artery. Eur $\mathrm{J}$ VascEndovascSurg 2000;19:579-586.

28. Gasecki AP, Eliasziw M, Ferguson GG, Hachinski V, Barnett HJM. Long-term prognosis and effect of endarterectomy in patients with symptomatic severe carotid stenosis and contralateral carotid stenosis or occlusion: results from NASCET. JNeurosurg 1995;83:778.

29. Park AE, McCarthy WJ, Pearce WH, Matsumura JS, Yao JS. Carotid plaque morphology correlates with presenting symptomatology. J VascSurg 1998;27:872-879.

30. Fisher M, Paganini-Hill A, Martin A, et al. Carotid plaque pathology: thrombosis, ulceration, and stroke pathogenesis. Stroke 2005;36:253-257.

31. Eliasziw M, Streifler JY, Fox AJ, Hachinski VC, Ferguson GG, Barnett HJ. Significance of plaque ulceration in symptomatic patients with high-grade carotid stenosis. North American Symptomatic Carotid Endarterectomy Trial. Stroke 1994;25:304-308.

32. Streifler JY, Eliasziw M, Fox AJ, et al. Angiographic detection of carotid plaque ulceration. Comparison with surgical observations in a multicenter study.North American Symptomatic Carotid Endarterectomy Trial. Stroke 1994;25:1130-1132.

33. Tartari S, Rizzati R, Righi R, et al. High-resolution MRI of carotid plaque with a neurovascular coil and contrast-enhanced MR angiography: one-stop shopping for the comprehensive assessment of carotid atherosclerosis. Am J Roentgenol 2011;196:1164-1171.

34. Takaya N, Yuan C, Chu B, et al. Association between carotid plaque characteristics and subsequent ischemic cerebrovascular events: a prospective assessment with MRI-initial results. Stroke 2006;37:818-823.

35. Morgenstern LB, Fox AJ, Sharpe BL, Eliasziw M, Barnett HJ, Grotta JC. The risks and benefits of carotid endarterectomy in patients with near occlusion of the carotid artery.North American Symptomatic Carotid Endarterectomy Trial (NASCET) Group. Neurology 1997;48:911-915.

36. Fox AJ, Eliasziw M, Rothwell PM, et al. Identification, prognosis, and management of patients with carotid artery near occlusion. AJNR Am J Neuroradiol 2005;26:2086-2094.

37. Hobson R, Weiss D, Fields W, Goldstone J, et al. Endarterectomy for asymptomatic carotid artery stenosis. Executive Committee for the Asymptomatic Carotid Atherosclerosis Study. JAMA 1995;273:1421-1428.

38. MRC Asymptomatic Carotid Surgery Trial Collaborative Group (2004) Prevention of disabling and fatal strokes by successful carotid endarterectomy in patients without recent neurological symptoms: randomised controlled trial. The Lancet. 363:1491-1502.

39. Tu JV, Wang H, Bowyer B, Green L, Fang J, Kucey D. Risk factors for death or stroke after carotid endarterectomy: observations from the Ontario Carotid Endarterectomy Registry. Stroke 2003;34:2568-2573.

40. Halliday A, Harrison M, Hayter E, et al. 10-year stroke prevention after successful carotid endarterectomy for asymptomatic stenosis (ACST-1): a multicentrerandomised trial. The Lancet 2010;376:1074-1084.

41. Chambers BR, Donnan GA. Carotid endarterectomy for asymptomatic carotid stenosis. Cochrane Database Syst Rev 2005: CD001923.

42. Barnett HJM, Meldrum HE, Eliasziw M. The appropriate use of carotid endarterectomy. CMAJ 2002;166:1169-1179.

43. Inzitari D, Eliasziw M, Gates P, et al. The causes and risk of stroke in patients with asymptomatic internal-carotid-artery stenosis. NEJM 2000;342:1693-1701. 
44. Goldstein LB, Samsa GP, Matchar DB, Oddone EZ. Multicenter review of preoperative risk factors for endarterectomy for asymptomatic carotid artery stenosis. Stroke 1998;29:750-753.

45. Abbott AL. Medical (nonsurgical) intervention alone is now best for prevention of stroke associated with asymptomatic severe carotid stenosis: results of a systematic review and analysis. Stroke 2009;40:e573-e583.

46. Spence JD, Tamayo A, Lownie SP, Ng WP, Ferguson GG. Absence of microemboli on transcranial doppler identifies lowrisk patients with asymptomatic carotid stenosis. Stroke 2005;36:2373-2378.

47. Spence JD, Coates V, Li H, et al. Effects of intensive medical therapy on microemboli and cardiovascular risk in asymptomatic carotid stenosis. Arch Neurol 2010;67:180-186.

48. Goldstein LB, Adams R, Alberts MJ, et al. Primary prevention of ischemic stroke: a guideline from the American Heart Association/American Stroke Association Stroke Council: cosponsored by the Atherosclerotic Peripheral Vascular Disease Interdisciplinary Working Group; Cardiovascular Nursing Council; Clinical Cardiology Council; Nutrition, Physical Activity, and Metabolism Council; and the Quality of Care and Outcomes Research Interdisciplinary Working Group: The American Academy of Neurology affirms the value of this guideline. Circulation 2006;113:1583-1633.

49. Taylor DW, Barnett HJM, Haynes RB, et al. Low-dose and high-dose acetylsalicylic acid for patients undergoing carotid endarterectomy: a randomised controlled trial. The Lancet 1999;353:2179-2184.

50. Engelter S, Lyrer P. Antiplatelet therapy for preventing stroke and other vascular events after carotid endarterectomy. Cochrane Database Syst Rev 2003:CD001458.

51. Poldermans D, Bax JJ, Kertai MD, et al. Statins are associated with a reduced incidence of perioperative mortality in patients undergoing major noncardiac vascular surgery. Circulation 2003;107:1848-1851.

52. Devereaux PJ, Beattie WS, Choi PTL, et al. How strong is the evidence for the use of perioperative beta blockers in non-cardiac surgery? Systematic review and meta-analysis of randomised controlled trials. BMJ 2005;331:313-321.

53. McGirt MJ, Perler BA, Brooke BS, et al. 3-Hydroxy-3-methylglutaryl coenzyme A reductase inhibitors reduce the risk of perioperative stroke and mortality after carotid endarterectomy. JVascSurg 2005;42:829-836.

54. Perler BA, Murphy K, Sternbach Y, Gailloud P, Shake JG. Immediate postoperative thrombolytic therapy: an aggressive strategy for neurologic salvage when cerebral thromboembolism complicates carotid endarterectomy. J VascSurg 2000;31:10331037.

55. Anzuini A, Briguori C, Roubin GS, et al. Emergency stenting to treat neurological complications occurring after carotid endarterectomy. J AmCollCardiol 2001;37:2074-2079.

56. Findlay JM, Marchak BE. Reoperation for acute hemispheric stroke after carotid endarterectomy: is there any value? Neurosurgery $2002 ; 50: 486-492$.
57. van Mook WN, Rennenberg RJ, Schurink GW, et al. Cerebral hyperperfusion syndrome. Lancet Neurol 2005;4:877-888.

58. Rothwell PM, Slattery J, Warlow CP. Clinical and angiographic predictors of stroke and death from carotid endarterectomy: systematic review. BMJ 1997;315:1571-1577.

59. AbuRahma AF, Jennings TG, Wulu JT, Tarakji L, Robinson PA. Redo carotid endarterectomy versus primary carotid endarterectomy. Stroke 2001;32:2787-2792.

60. Metzger DC. Embolic protection in carotid artery stenting: new options. TechVascIntervRadiol 2011;14:86-94.

61. Bates ER, Babb JD, Casey DE, et al. ACCF/SCAI/SVMB/SIR/ ASITN 2007 clinical expert consensus document on carotid stenting. J Am CollCardiol 2007;49:126-170.

62. Yadav JS, Wholey MH, Kuntz RE, et al. Protected carotid-artery stenting versus endarterectomy in high-risk patients. $\mathrm{N}$ Engl $\mathrm{J}$ Med 2004;351:1493-1501.

63. Gurm HS, Yadav JS, Fayad P, et al. Long-term results of carotid stenting versus endarterectomy in high-risk patients. NEJM 2008;358:1572-1579.

64. Goodney PP, Powell RJ. Carotid artery stenting: what have we learned from the clinical trials and registries and where do we go from here. Ann Vasc Surg 2008;22:148-158.

65. SPACE, Collaborative Group. 30 day results from the SPACE trial of stent-protected angioplasty versus carotid endarterectomy in symptomatic patients: a randomised non-inferiority trial. The Lancet 2006;368:1239-1247.

66. Eckstein H-H, Ringleb P, Allenberg J-R, et al. Results of the StentProtected Angioplasty versus Carotid Endarterectomy (SPACE) study to treat symptomatic stenoses at 2 years: a multinational, prospective, randomised trial. The Lancet Neurology 2008;7:893-902.

67. Mas J-L, Chatellier G, Beyssen B, et al. Endarterectomy versus stenting in patients with symptomatic severe carotid stenosis. N Engl J Med 2006;355:1660-1671.

68. Gray WA, Rosenfield KA, Jaff MR, Chaturvedi S, Peng L, Verta P. Influence of site and operator characteristics on carotid artery stent outcomes: analysis of the CAPTURE 2 (Carotid ACCULINK/ ACCUNET Post Approval Trial to Uncover Rare Events) Clinical Study. JACCCardiovascInterv 2011;4:235-246.

69. Harjai KJ, Mehta RH. Trials and tribulations of carotid artery stenting: the interventionalists' perspective on SAPPHIRE, EVA3S, and SPACE trials. J IntervCardiol 2007;20:389-394.

70. Howard VJ, Lutsep HL, Mackey A, et al. Influence of sex on outcomes of stenting versus endarterectomy: a subgroup analysis of the Carotid Revascularization Endarterectomy versus Stenting Trial (CREST). The Lancet Neurol 2011;10:530-537.

71. Economopoulos KP, Sergentanis TN, Tsivgoulis G, Mariolis AD, Stefanadis C. Carotid artery stenting versus carotid endarterectomy: a comprehensive meta-analysis of short-term and long-term outcomes. Stroke 2011;42:687-692.

72. Bangalore S, Kumar S, Wetterslev J, et al. Carotid artery stenting vs carotid endarterectomy: meta-analysis and diversity-adjusted trial sequential analysis of randomized trials. Arch Neurol 2011;68:172-184. 